\section{Is it currently reasonable to offer short, 14-day antibiotic therapies after a surgical synovectomy in native joint septic arthritis?}

Gjika et al ${ }^{1}$ recently reported the non-inferiority of 2 -week versus 4-week antibiotic therapy after systematic surgical washing (with or without synovectomy) in the management of septic arthritis. Although this randomised, controlled trial adds important new insights to the management of patients with infectious arthritis, it has several drawbacks that limit generalisation of the results.

First, the patients included in this study were not representative of a usual population of native joint septic arthritis patients. These patients had mainly septic arthritis affecting the small joints (metatarsophalangeal, metacarpophalangeal and proximal interphalangeal joints in $85 \%$ of cases). Moreover, in most cases, contamination occurred after direct inoculation following skin invasion (bite/scratch or post-traumatic), with only $4 \%$ of patients with systemic signs of infection. Finally, patients were included on the basis of having been treated with surgical drainage, the indications for which are largely centre dependent. These characteristics are probably explained by the monocentric design of the study. Native joint septic arthritis affects mainly large joints, such as the knees, ankles or hips in more than $70 \%$ of the cases. Moreover, haematogenous seeding is by far the most frequent type of inoculation (95\%), with concomitant bacteraemia occurring in about $50 \%$ of the cases. ${ }^{2-4}$ The joints involved, as well as the bacterial species found in this study, clearly differ from those observed following systemic contamination: $50 \%$ of the pathogens were Gram-negative rods (25\%) (with $>10 \%$ Pasteurella sp.) or Streptococcus sp. (25\%), with less than one-third the result of staphylococcal infection. This bacterial distribution is not representative of the type of septic arthritis either referred to emergency departments or managed in rheumatology/infectious disease departments. ${ }^{2-4}$ Importantly, the bacterial inoculum and virulence factors associated with haematogenous inoculation are very different from those found in direct inoculation through a bite or direct skin trauma. Underrepresentation of the bacteria capable of persistence and slow metabolism activity, such as Staphylococcus aureus, which more readily lead to relapses, may have artificially explained the noninferiority and very high rate of cure without relapse, despite the short-term antibiotherapy observed by the authors. Moreover, the median final assessment was 2 months, while the classic, gold-standard definition of recovery without relapse in osteoarticular infections is 1 year. ${ }^{5}$ The authors reported three relapses (two of which occurred with $S$. aureus infection) over this short period of systematic follow-up. Few results are available on the radiological and functional evolution of the patients (one-third of the patients had follow-up X-ray) in the midterm and long term. This is even more important knowing the high prevalence of structural damage and functional sequelae reported in prospective series. $^{6}$

From a methodological point of view, the authors calculated that 48 patients needed to be included in each arm, with a noninferiority margin of $10 \%$. This low number of subjects required was based on the hypothesis of a high healing goal of $96 \%$ at 2 months. If we consider a 1 -year cure of $90 \%$, closer to the objectives of other large, non-inferiority trials on antibiotic treatment of osteoarticular infections, ${ }^{57}$ with the same margin of error at $10 \%$ and the same power $(1-\beta)$ of $80 \%$, the number of patients needed would have been 112 patients in each group. In an ongoing study comparing 3 weeks versus 6 weeks of antibiotic therapy in native septic joint arthritis (SHASAR, NCT0371692), the number of subjects required to demonstrate non-inferiority in the per-protocol analysis, with a 5\% loss of patients included during follow-up, was calculated as 175 patients in each group. ${ }^{8}$ In addition, the authors did not explain why the number of subjects included (77 in each arm) was much higher than planned. Finally, subgroup analyses were performed in patients with hand and wrist involvement, although this was not initially planned (not declared on clinicaltrial.gov). Furthermore, these subgroup analyses were the only ones presented in the protocol population. It would have been interesting to report the results for the primary endpoint in the whole per-protocol population, as recommended in a non-inferiority study, to avoid the risk of overestimating efficacy, and not just present the results for hand and wrist involvement. ${ }^{9}$

Overall, even if these results are of great interest in an era of decreasing antibiotic treatment duration for ecological and economic reasons, it seems difficult to generalise and transpose the results of this study to daily practice for the management of native joint septic arthritis, and the conclusions drawn from this trial must be limited to patients with small joint infection caused by direct inoculation.

Guillaume Coiffier $\odot,^{1,2}$ Marion Couderc $\odot,,^{2,3}$ Raphaele Seror, ${ }^{2,4}$
Geraldine Bart, ${ }^{1,2}$ Christelle Darrieutort-Laffite $\odot,{ }^{2,5,6}$ Benoit Le Goff $\odot^{2,5}$
${ }^{1}$ Rhumatologie, CHU de Rennes, Rennes, France
${ }^{2}$ Osteoarticular Infections Working Group of the French Society of Rheumatology,
Paris, France
${ }^{3}$ Rheumatology, CHU Gabriel Montpied, Clermont-Ferrand, France
${ }^{4}$ Rheumatology, Hôpitaux Universitaires Paris-Sud, Le Kremlin Bicêtre, France
${ }^{5}$ Rheumatology, CHU Nantes, Nantes, France
${ }^{6}$ INSERM UMR1238, Nantes, France

Correspondence to Dr Guillaume Coiffier, Rhumatologie, CHU de Rennes, Rennes 35000, France; guillaume.coiffier@chu-rennes.fr

Contributors All authors contributed to the argument and writing of this correspondence response.

Funding The authors have not declared a specific grant for this research from any funding agency in the public, commercial or not-for-profit sectors.

Competing interests None declared.

Patient consent for publication Not required.

Provenance and peer review Not commissioned; internally peer reviewed.

(c) Author(s) (or their employer(s)) 2020. No commercial re-use. See rights and permissions. Published by BMJ.

$$
\text { Check for updates }
$$

To cite Coiffier G, Couderc M, Seror R, et al. Ann Rheum Dis 2020;79:e146.

Received 12 June 2019

Accepted 17 June 2019

Published Online First 5 July 2019

\section{S Linked}

- http://dx.doi.org/10.1136/annrheumdis-2019-215905

Ann Rheum Dis 2020;79:e146. doi:10.1136/annrheumdis-2019-215887

\section{ORCID iDs}

Guillaume Coiffier http://orcid.org/0000-0003-3560-128X

Marion Couderc http://orcid.org/0000-0002-2001-1132

Christelle Darrieutort-Laffite http://orcid.org/0000-0002-1871-0073

Benoit Le Goff http://orcid.org/0000-0002-4540-4549

\section{REFERENCES}

1 Gjika E, Beaulieu J-Y, Vakalopoulos K, et al. Two weeks versus four weeks of antibiotic therapy after surgical drainage for native joint bacterial arthritis: a prospective, randomised, 
non-inferiority trial. Ann Rheum Dis 2019. doi:10.1136/annrheumdis-2019-215116. [Epub ahead of print: 16 Apr 2019].

2 Mathews $\mathrm{CJ}$, Weston VC, Jones A, et al. Bacterial septic arthritis in adults. The Lancet 2010;375:846-55.

3 Clerc 0, Prod'hom G, Greub G, et al. Adult native septic arthritis: a review of 10 years of experience and lessons for empirical antibiotic therapy. J Antimicrob Chemother 2011;66:1168-73.

4 Dubost J-J, Couderc M, Tatar Z, et al. Three-decade trends in the distribution of organisms causing septic arthritis in native joints: single-center study of 374 cases. Joint Bone Spine 2014:81:438-40.
5 Bernard L, Dinh A, Ghout I, et al. Antibiotic treatment for 6 weeks versus 12 Weeks in patients with pyogenic vertebral osteomyelitis: an open-label, non-inferiority, randomised, controlled trial. Lancet 2015;385:875-82.

6 Gaigneux E, Cormier G, Varin S, et al. Ultrasound abnormalities in septic arthritis are associated with functional outcomes. Joint Bone Spine 2017;84:599-604.

7 Li H-K, Rombach I, Zambellas R, et al. Oral versus intravenous antibiotics for bone and joint infection. N Engl J Med 2019;380:425-36.

8 Short 3-week Antibiotique Treatment versus 6 weeks in Adults with Septic Arthritis of Native Joint (SHASAR). 2018 ClinicalTrials.gov NCT03716921.

9 Dunn DT, Copas AJ, Brocklehurst P. Superiority and non-inferiority: two sides of the same coin? Trials 2018;19. 